\title{
Chromospheric variabilities of $M$ active stars based on Guoshoujing Telescope
}

\author{
Q. F. Pi ${ }^{1}$, L. Y. Zhang ${ }^{1}$, J. R. Shi ${ }^{2}$, H. $\mathbf{W u}^{2}$, Y. H. Zhao ${ }^{2}$, A. L. Luo ${ }^{2}$, \\ J. K. Zhao ${ }^{2}$, A. Y. Zhou ${ }^{2}$, X. S. Fang ${ }^{1}$ and Lamost Collaboration ${ }^{2}$ \\ ${ }^{1}$ College of Science/Department of Physics, Guizhou University, Guiyang 550025, China \\ email: Liy_zhang@hotmail.com \\ ${ }^{2}$ National Astronomical Observatories, Chinese Academy of Sciences, Beijing 100012, China
}

\begin{abstract}
For the M active catalogue of Guoshoujing Telescope (LAMOST), 933 sources are presented in at least two exposures. We found that many $\mathrm{M}$ active stars show chromospheric variability in the $\mathrm{Ca}$ II $\mathrm{H}, \mathrm{H}_{\alpha}, \mathrm{H}_{\beta}$, and $\mathrm{H}_{\gamma}$ lines on short or long timescales.
\end{abstract}

Keywords. stars: M, stars: activity, stars: chromospheres, stars: spectra

Chromospheric variability of $\mathrm{M}$ dwarf stars are very interesting (Hilton et al. 2012; Kruse et al. 2012; etc). We found $6391 \mathrm{M}$ active candidates (Zhang et al. 2012, 2013) from LAMOST spectral survey (Cui et al. 2012; Zhao et al. 2012; Luo et al. 2012; Deng et al. 2012). 933 sources are found in at least two exposures, in which 193 with more than 3 three exposures. These spectra, obtained at different times, can be used to examine chromospheric variability. We measured the equivalent widths of the $\mathrm{Ca} \mathrm{II} \mathrm{H}, \mathrm{H}_{\alpha}, \mathrm{H}_{\beta}$, and $\mathrm{H}_{\gamma}$ lines using the program of Hawley et al. (2002) and West et al. (2011). The wavelength regions used for the continuum and line regions are similar to that of Hilton et al. (2012). We found that chromospheric activity of most active stars had changed on either short or long time scales. Figure 1 shows an example for LAMOST J045556.06+303620.6 in Ca II $\mathrm{H}, \mathrm{H}_{\alpha}, \mathrm{H}_{\beta}$, and $\mathrm{H}_{\gamma}$ lines.
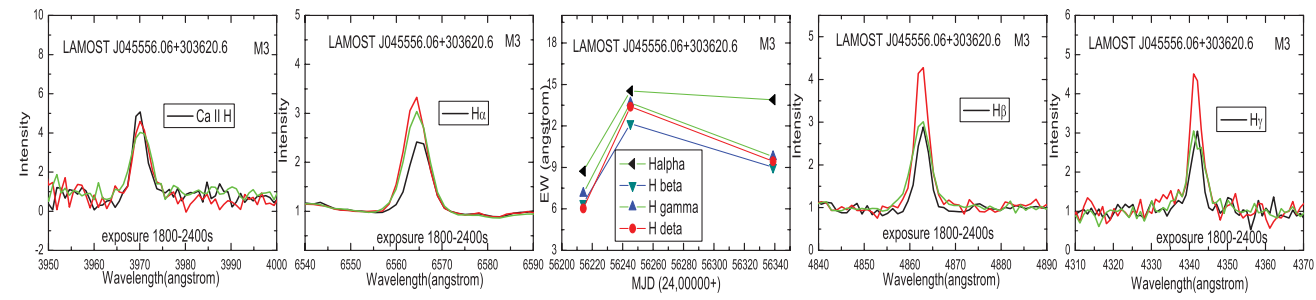

Figure 1. The spectra variation and EWs light curves of LAMOST J045556.06+303620.6.

Acknowledgements This work is supported by the NSFC of No 10978010 and 11263001 .

\section{References}

Cui X. Q., Zhao Y. H., Chu Y. Q., et al., 2012, RAA, 12, 1197

Hilton E. J., West A. A., Hawley S. L., et al., 2010, AJ, 140, 1402

Hawley S. L., Covey K. R., Knapp G. R., et al., 2002, AJ, 123, 3409

Luo A. L., Zhang H. T., Zhao, Y. H., Zhao G., et al., 2012, RAA, 12, 1243

Kruse E. A., Berger E., Knapp G. R., et al., 2010, AJ, 722, 1352

West A. A., Morgan D. P., Bochanski J. J., et al., 2011, AJ, 141, 97

Zhang L. Y., Shi J. R., Zhao J. K., Luo A. L., et al., 2012, IAU294, 209

Zhang L. Y., Zhang G. Y., Shi J. R., Luo A. L., et al., 2013, IAU298,in print

Zhao G., Zhao Y. H., Chu Y. Q., et al., 2012, RAA, 12, 723 